\title{
On the Zeros of the Riemann Zeta Function in the Critical Strip. III
}

\author{
By J. van de Lune and H. J. J. te Riele
}

\begin{abstract}
We describe extensive computations which show that Riemann's zeta function $\zeta(s)$ has exactly $300,000,001$ zeros of the form $\sigma+i t$ in the region $0<t<119,590,809.282$. All these zeros are simple and lie on the line $\sigma=1$. (This extends a similar result for the first 200,000,001 zeros, established by Brent, van de Lune, te Riele and Winter in Math. Comp., v. 39, 1982, pp. 681-688.) Counts of the numbers of Gram blocks of various types and the failures of "Rosser's rule" are given, together with some graphs of the function $Z(t)$ near the first observed failures of Rosser's rule.
\end{abstract}

1. Introduction. This paper is a continuation of Brent [1] and Brent, van de Lune, te Riele and Winter [2]. We have extended the computations described there to show that the first 300,000,001 zeros of Riemann's zeta function $\zeta(s)$ in the critical strip are simple and lie on the line $\sigma=\frac{1}{2}$. After separating the zeros of $Z(t)$ in the range $\left[g_{200,000,000}, g_{300,000,000}\right)$ with a slightly changed version of the FORTRAN/COMPASS program described in van de Lune, te Riele and Winter [4], we ran the computation a little further and found four Gram blocks (of length 1) in $\left[g_{300,000,000}, g_{300,000,004}\right)$. By applying Theorem 3.2 of [1], we completed the proof of our claim.

A complete listing of our program may be found in van de Lune and te Riele [5]. We also refer to [4] and [5] for a more detailed description of our slightly adapted strategy (compared with Brent's) for finding the required number of sign changes of $Z(t)$ in Gram blocks of length $\geqslant 2$. It may be noted here that the average number of $Z$-evaluations needed to separate two successive zeros of $Z(t)$ varied slightly around 1.19. Comparing this with our previous computations, it appears that the oscillatory behavior of $Z(t)$ has not changed very much.

Besides 126 exceptions to Rosser's rule of length 2, we also found three exceptions of length 3. The latter ones appeared as Gram blocks of length 3 containing only 1 zero, followed or preceded by a Gram block of length 1 with 3 zeros. More details are given in the next section, including some graphs of $Z(t)$ in the neighborhood of various types of exceptions to Rosser's rule which have emerged. (Previously, two exceptions of length 3 were implicitly given by Karkoschka and Werner [3], viz., $B_{1,089,751,985}$ and $B_{10,008,051,629}$, although these are far beyond the range covered by our systematic search.) In the next section we also give a number of tables which are (nearly) similar to those given in [1] and [2].

We intend to extend our computations in the near future.

Received February 8, 1983.

1980 Mathematics Subject Classification. Primary 10H05; Secondary 10-04, 65E05, 30-04.

Key words and phrases. Gram blocks, Riemann hypothesis, Riemann zeta function, Rosser's rule. 
2. Statistics. The counts given here are now exact (in contrast with the LR \& $\mathrm{W}$-counts given in [2]). This was realized by immediately computing $Z_{B}\left(g_{n}\right)$ in case $\left|Z_{A}\left(g_{n}\right)\right|$ was too small (rather than computing $Z_{A}\left(g_{n}-\delta\right)$ for a few small values of $\delta$, as we did in [2]). We never met a value of $t$ for which our method $B$ could not determine the sign of $Z(t)$ rigorously, with the bounds given in Section 3 of [2].

\section{TABLE 1}

The 129 exceptions to Rosser's rule in $\left[g_{200,000,000}, g_{300,000,000}\right)$

126 of length 2 . Notation: $n$ (type) extreme $S(t)$,

where $n$ is the index of the Gram block

$B_{n}=\left[g_{n}, g_{n+2}\right)$ with zero-pattern " 00 "

$\begin{array}{lr}201007375(1) & -2.002900 \\ 201030606(2) & 2.111895 \\ 201184291(2) & 2.001518 \\ 201685414(5) & -2.016715 \\ 202762876(2) & 2.011439 \\ 202860958(2) & 2.018888 \\ 203832578(2) & 2.063611 \\ 205880544(1) & -2.017679 \\ 206357111(1) & -2.031216 \\ 207159768(2) & 2.033954 \\ 207167344(2) & 2.029320 \\ 207669541(2) & 2.020740 \\ 208053426(1) & -2.073357 \\ 208110028(2) & 2.031212 \\ 209513827(2) & 2.023920 \\ 212623522(1) & -2.010194 \\ 213841715(1) & -2.024334 \\ 214012333(1) & -2.010937 \\ 214073567(1) & -2.009287 \\ 215170601(2) & 2.007728 \\ 215881040(2) & 2.021267 \\ 216274605(2) & 2.052279 \\ 216957121(2) & 2.032421 \\ 217323208(1) & -2.013607 \\ 218799264(1) & -2.040304 \\ 218803558(2) & 2.013448 \\ 219735146(1) & -2.026815 \\ 219830063(2) & 2.015232 \\ 219897904(1) & -2.081132 \\ 221205545(1) & -2.014535 \\ 223601929(1) & -2.101580 \\ 223907077(2) & 2.007094 \\ 223970397(1) & -2.028754 \\ 224874046(6) & 2.022804 \\ 225291157(1) & -2.152675 \\ 227481734(1) & -2.018298 \\ 228006443(2) & 2.023042 \\ 228357900(1) & -2.022758 \\ 228386399(1) & -2.008899 \\ 228907446(1) & -2.018338 \\ 228984553(2) & 2.032004 \\ 229140286(2) & 2.000109\end{array}$

$\begin{array}{lr}231810024(1) & -2.026611 \\ 232838063(2) & 2.022488 \\ 234389089(2) & 2.106429 \\ 235588194(1) & -2.001915 \\ 236645695(1) & -2.089639 \\ 236962877(2) & 2.023259 \\ 237516725(4) & 2.108817 \\ 240004911(1) & -2.000249 \\ 240221307(2) & 2.096293 \\ 241549003(1) & -2.036151 \\ 241729717(1) & -2.025503 \\ 241743685(2) & 2.070155 \\ 243780201(2) & 2.025648 \\ 243801317(1) & -2.020358 \\ 244122072(1) & -2.035325 \\ 244691225(2) & 2.018927 \\ 244841577(1) & -2.053021 \\ 245813461(1) & -2.035731 \\ 246299475(1) & -2.001039 \\ 246450177(2) & 2.116655 \\ 249069349(1) & -2.020698 \\ 250076378(1) & -2.036397 \\ 252442158(2) & 2.085094 \\ 252904232(2) & 2.112235 \\ 255145220(1) & -2.002286 \\ 255285972(2) & 2.034861 \\ 256713230(1) & -2.015377 \\ 257992082(1) & -2.042307 \\ 258447957(6) & 2.005655 \\ 259298046(2) & 2.091955 \\ 262141503(1) & -2.006009 \\ 263681744(2) & 2.006016 \\ 266617122(1) & -2.046423 \\ 266628045(2) & 2.048158 \\ 267305763(1) & -2.028836 \\ 267388405(2) & 2.012716 \\ 267441673(2) & 2.085691 \\ 267464886(1) & -2.006418 \\ 267554908(2) & 2.112706 \\ 269787480(1) & -2.080890 \\ 270881434(1) & -2.026487 \\ 270997584(2) & 2.021752\end{array}$

$\begin{array}{lr}272096379(2) & 2.001554 \\ 272583009(1) & -2.032037 \\ 274190882(2) & 2.008416 \\ 274268747(1) & -2.018420 \\ 275297430(2) & 2.014738 \\ 275545477(2) & 2.032087 \\ 275898480(2) & 2.005068 \\ 275953000(1) & -2.007296 \\ 277117197(5) & -2.069283 \\ 277447311(2) & 2.058999 \\ 279059658(2) & 2.037126 \\ 279259145(2) & 2.033129 \\ 279513637(2) & 2.000375 \\ 279849070(2) & 2.048163 \\ 280291419(1) & -2.021221 \\ 281449426(2) & 2.000609 \\ 281507954(2) & 2.001841 \\ 281825600(1) & -2.033191 \\ 282547094(2) & 2.002833 \\ 283120964(2) & 2.028096 \\ 283323493(1) & -2.032511 \\ 284764536(2) & 2.001422 \\ 286172640(2) & 2.042925 \\ 286688824(1) & -2.046407 \\ 287222173(2) & 2.048065 \\ 287235535(2) & 2.024894 \\ 287304862(2) & 2.003208 \\ 287433571(1) & -2.021945 \\ 287823551(1) & -2.038399 \\ 287872423(2) & 2.016959 \\ 288766616(2) & 2.024072 \\ 290122964(2) & 2.039001 \\ 290450849(5) & -2.068090 \\ 291426142(2) & 2.075533 \\ 292810354(2) & 2.048278 \\ 293109862(2) & 2.013978 \\ 293398055(2) & 2.042772 \\ 294134427(2) & 2.043302 \\ 294216438(1) & -2.005490 \\ 295367142(2) & 2.049246 \\ 297834112(2) & 2.022351 \\ 299099970(2) & 2.030191\end{array}$

$272096379(2) \quad 2.001554$ $272583009(1)-2.032037$ $274190882(2) \quad 2.008416$ $274268747(1)-2.018420$ $275545477(2) \quad 2.032087$ $275898480(2) \quad 2.005068$ $275953000(1)-2.007296$ $277117197(5)-2.069283$ $277447311(2) \quad 2.058999$ $279059658(2) \quad 2.037126$ $279259145(2) \quad 2.033129$ $279513637(2) \quad 2.000375$ $279849070(2) \quad 2.048163$ $280291419(1)-2.021221$ $281449426(2) \quad 2.000609$ $281507954(2) \quad 2.001841$ $281825600(1)-2.033191$ $282547094(2) \quad 2.002833$ $283120964(2) \quad 2.028096$ $286172640(2) \quad 2.042925$ $286688824(1)-2.046407$ $287222173(2) \quad 2.048065$ $287235535(2) \quad 2.024894$ $287304862(2) \quad 2.003208$ $287433571(1)-2.021945$ $287823551(1)-2.038399$ $287872423(2) \quad 2.016959$ $288766616(2) \quad 2.024072$ $290122964(2) \quad 2.039001$ $290450849(5)-2.068090$ $291426142(2) \quad 2.075533$ $292810354(2) \quad 2.048278$ $293109862(2) \quad 2.013978$ $293398055(2) \quad 2.042772$ $295367142(2) \quad 2.049246$ $299099970(2) \quad 2.030191$

3 of length 3. Notation: $n$ (type) extreme $S(t)$,

where $n$ is the index of the Gram block

$B_{n}=\left[g_{n}, g_{n+3}\right)$ with zero-pattern " 010 "

$207482540(2) 2.000431 \quad 241389213(1)-2.010430 \quad 266527881(2)-2.008550$

(For the definition of the types in case of length 3 -exceptions, see Table 2.) 
TABLE 2

Various types of exceptions to Rosser's rule and their frequencies in $\left[g_{200,000,000}, g_{300.000 .000}\right)$
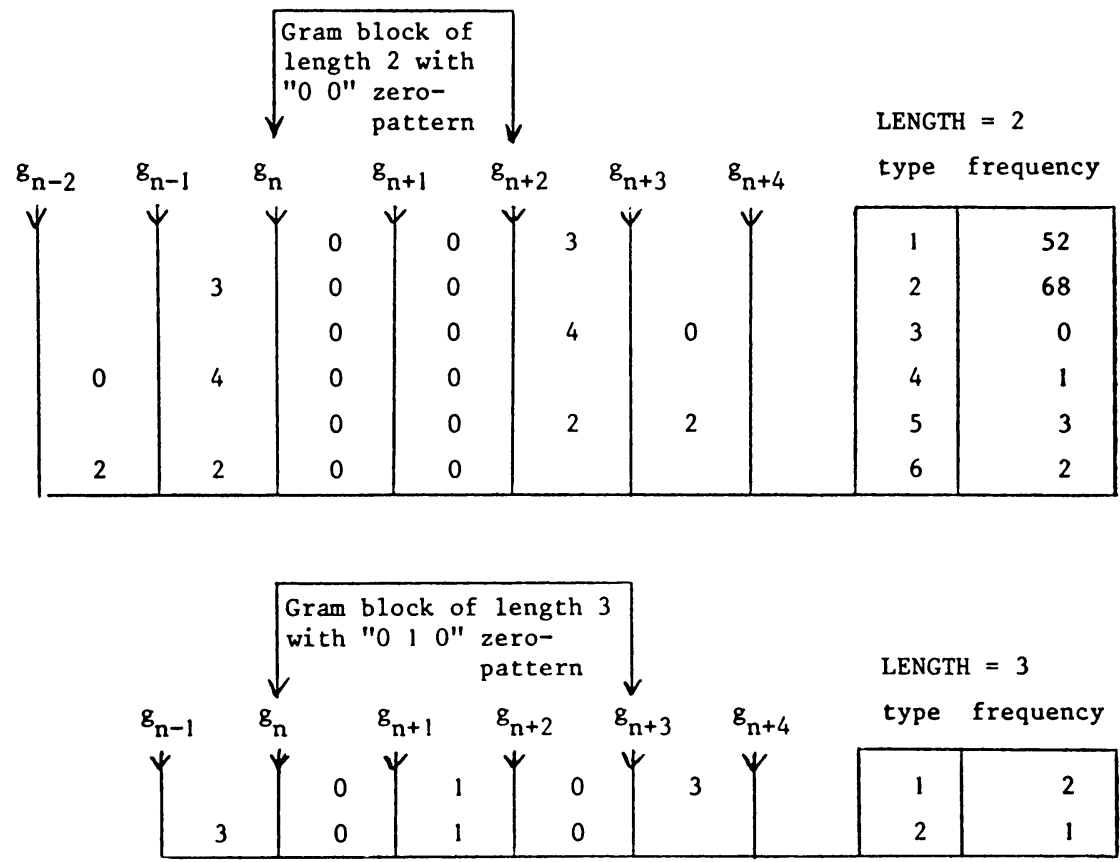

TABLE 3

Number of Gram blocks of given length

$J^{\prime}(k, n):=J\left(k, n+10^{7}\right)-J(k, n)$

\begin{tabular}{|c|c|c|c|c|c|c|c|c|}
\hline $\mathrm{n}$ & $J^{\prime}(1, n)$ & $J^{\prime}(2, n)$ & $\mathrm{J}^{\prime}(3, \mathrm{n})$ & $J^{\prime}(4, n)$ & $J^{\prime}(5, n)$ & $J^{\prime}(6, n) J^{\prime}$ & $(7, n) J^{\prime}(8$ & $8, n)$ \\
\hline $200,000,000$ & $6,973,019$ & $1,056,242$ & 236,180 & 44,997 & 4,838 & 272 & 21 & 0 \\
\hline $210,000,000$ & $6,971,273$ & $1,055,810$ & 236,438 & 45,249 & 4,976 & 297 & 17 & 2 \\
\hline $220,000,000$ & $6,966,636$ & $1,056,779$ & 236,573 & 45,864 & 4,921 & 312 & 22 & 0 \\
\hline $230,000,000$ & $6,965,176$ & $1,056,494$ & 236,663 & 46,250 & 4,979 & 295 & 26 & 0 \\
\hline $240,000,000$ & $6,961,469$ & $1,057,120$ & 237,279 & 46,150 & 5,156 & 327 & 16 & 0 \\
\hline $250,000,000$ & $6,957,609$ & $1,057,612$ & 237,551 & 46,633 & 5,145 & 341 & 29 & 1 \\
\hline $260,000,000$ & $6,955,568$ & $1,056,920$ & 238,418 & 46,694 & 5,300 & 306 & 30 & 2 \\
\hline $270,000,000$ & $6,951,895$ & $1,057,635$ & 238,483 & 47,211 & 5,257 & 353 & 20 & 0 \\
\hline $280,000,000$ & $6,950,241$ & $1,056,940$ & 238,974 & 47,373 & 5,420 & 366 & 24 & 0 \\
\hline $290,000,000$ & $6,948,974$ & $1,058,297$ & 238,885 & 47,295 & 5,254 & 354 & 29 & 0 \\
\hline Totals & $69,601,860$ & $10,569,849$ & $2,375,444$ & 463,716 & 51,246 & 3,223 & 234 & 5 \\
\hline
\end{tabular}


TABLE 4

Number of Gram intervals containing exactly $m$ zeros

\begin{tabular}{|c|c|c|c|c|c|c|}
\hline $\mathrm{n}$ & $\mathrm{m}=0$ & $\mathrm{~m}=1$ & $\mathrm{~m}=2$ & $\mathrm{~m}=3$ & $\mathrm{~m}=$ & $=4$ \\
\hline $200,000,000$ & $1,360,273$ & $7,297,177$ & $1,324,827$ & 17,723 & 0 & 0 \\
\hline $210,000,000$ & $1,360,333$ & $7,296,878$ & $1,325,245$ & 17,544 & 0 & 0 \\
\hline $220,000,000$ & $1,362,066$ & $7,293,463$ & $1,326,876$ & 17,595 & 0 & 0 \\
\hline $230,000,000$ & $1,362,561$ & $7,292,733$ & $1,326,852$ & 17,853 & 1 & 1 \\
\hline $240,000,000$ & $1,363,647$ & $7,290,305$ & $1,328,449$ & 17,599 & 0 & 0 \\
\hline $250,000,000$ & $1,365,010$ & $7,287,678$ & $1,329,614$ & 17,698 & 0 & \\
\hline $260,000,000$ & $1,365,698$ & $7,286,632$ & $1,329,642$ & 18,028 & 0 & 0 \\
\hline $270,000,000$ & $1,366,741$ & $7,284,300$ & $1,331,176$ & 17,783 & 0 & 0 \\
\hline $280,000,000$ & $1,367,037$ & $7,283,866$ & $1,331,158$ & 17,939 & 0 & \\
\hline $290,000,000$ & $1,367,806$ & $7,282,080$ & $1,332,422$ & 17,692 & 0 & 0 \\
\hline Totals & $13,641,172$ & $72,895,112$ & $13,286,261$ & 177,454 & 1 & \\
\hline
\end{tabular}

TABLE 5

Number of Gram blocks of type $(j, k), 1 \leqslant j \leqslant 8,1 \leqslant k \leqslant j$,

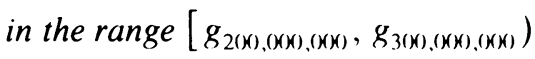

\begin{tabular}{|c|c|c|c|c|c|c|c|c|}
\hline$+j$ & $\begin{array}{c}\mathrm{k} \rightarrow \\
1\end{array}$ & 2 & 3 & 4 & 5 & 6 & 78 & Totals \\
\hline 1 & $69,601,860$ & & & & & & & $69,601,860$ \\
\hline 2 & $\begin{array}{r}5,285,566 \\
(50)\end{array}$ & $\begin{array}{r}5,284,152 \\
(50)\end{array}$ & $\begin{array}{rrr}126 & 0 \\
5 & 0 & 0\end{array}$ & $\begin{array}{l}\text { - blocks } \\
\text { - blocks }\end{array}$ & & & & $10,569,849$ \\
\hline 3 & $\begin{array}{r}1,125,098 \\
(47)\end{array}$ & $\begin{array}{r}124,535 \\
(5)\end{array}$ & $\begin{array}{r}1,125,808 \\
(47)\end{array}$ & $\begin{array}{llll}3 & 0 & 1 & 0 "\end{array}$ & - blocks & & & $2,375,444$ \\
\hline 4 & $\begin{array}{r}212,286 \\
(46)\end{array}$ & $\begin{array}{r}19,862 \\
(4)\end{array}$ & $\begin{array}{r}19,649 \\
(4)\end{array}$ & $\begin{array}{r}211,919 \\
(46)\end{array}$ & & & & 463,716 \\
\hline 5 & $\begin{array}{c}20,326 \\
(40)\end{array}$ & $\begin{array}{r}4,252 \\
(8)\end{array}$ & $\begin{array}{r}2,177 \\
(4)\end{array}$ & $\begin{array}{r}4,346 \\
(8)\end{array}$ & $\begin{array}{r}20,145 \\
(39)\end{array}$ & & & 51,246 \\
\hline 6 & $\begin{array}{l}488 \\
(15)\end{array}$ & $\begin{array}{r}866 \\
(27)\end{array}$ & $\begin{array}{l}263 \\
(8)\end{array}$ & $\begin{array}{l}286 \\
(9)\end{array}$ & $\begin{array}{r}859 \\
(27)\end{array}$ & $\begin{array}{r}461 \\
(14)\end{array}$ & & 3,223 \\
\hline 7 & $\left.2^{\star}\right)$ & 13 & 42 & 9 & 40 & 67 & $\left.1^{\star}\right)$ & 234 \\
\hline 8 & 0 & 0 & 2 & 0 & 0 & 3 & 00 & 5 \\
\hline
\end{tabular}

${ }^{\star}$ viz. $B_{n}$, for $n=258,779,892,282,307,390$ and $299,608,968$.

In Table 1 we present a list of the 126 exceptions to Rosser's rule of length 2 and the 3 of length 3 , in the range $\left[g_{200,000,000}, g_{300,000,000}\right)$, including their types and the local extreme values of $S(t)$. Note the occurrence of a type 4-exception (the first one observed) which yields the second Gram interval with four zeros (viz. $G_{237,516,724}$; the first one is $\left.G_{61,331,768}[1]\right)$.

Table 2 gives a survey of the various types of exceptions to Rosser's rule, observed until now, and their frequencies in $\left[g_{200,000,000}, g_{300,000,000}\right)$. 

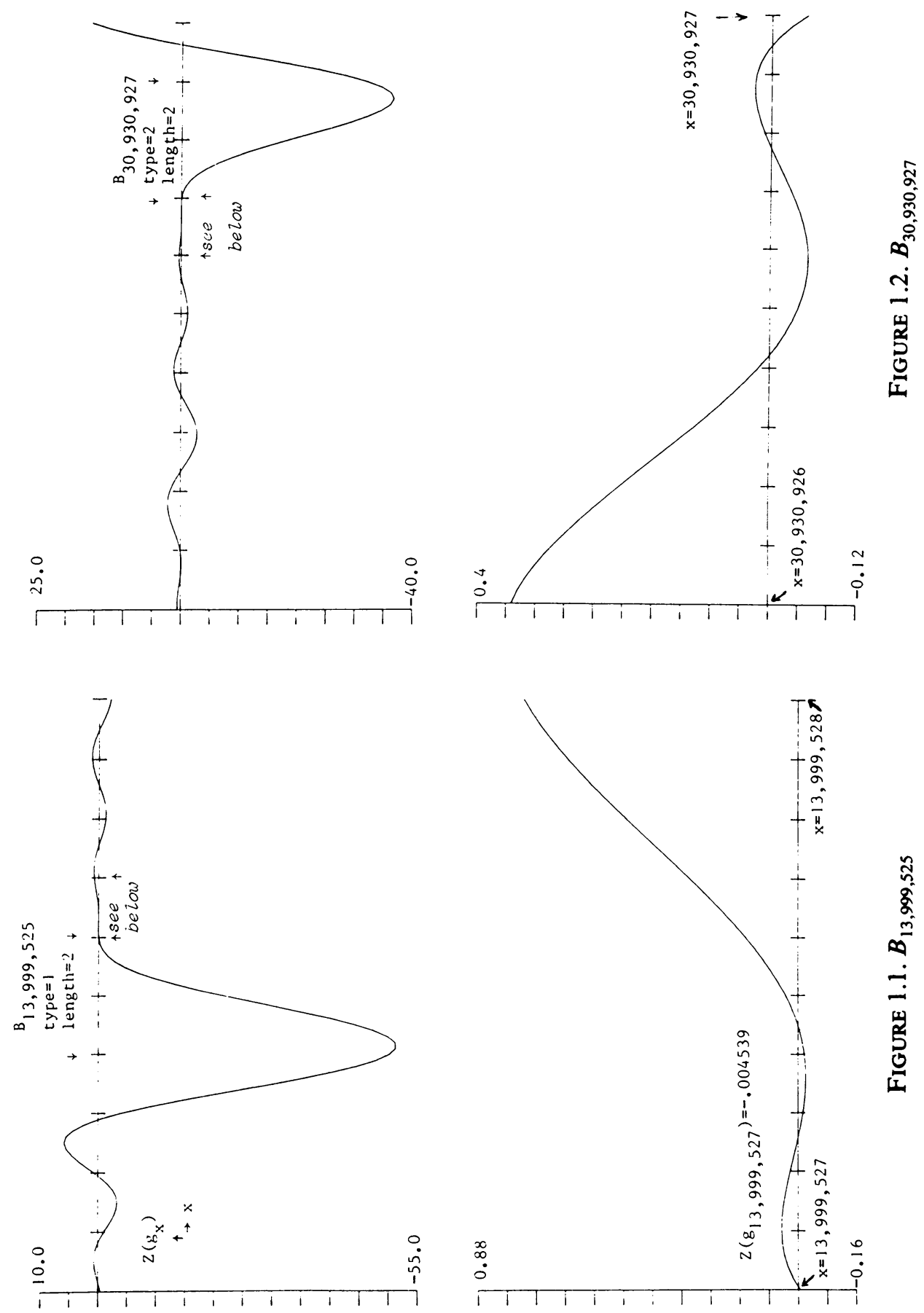

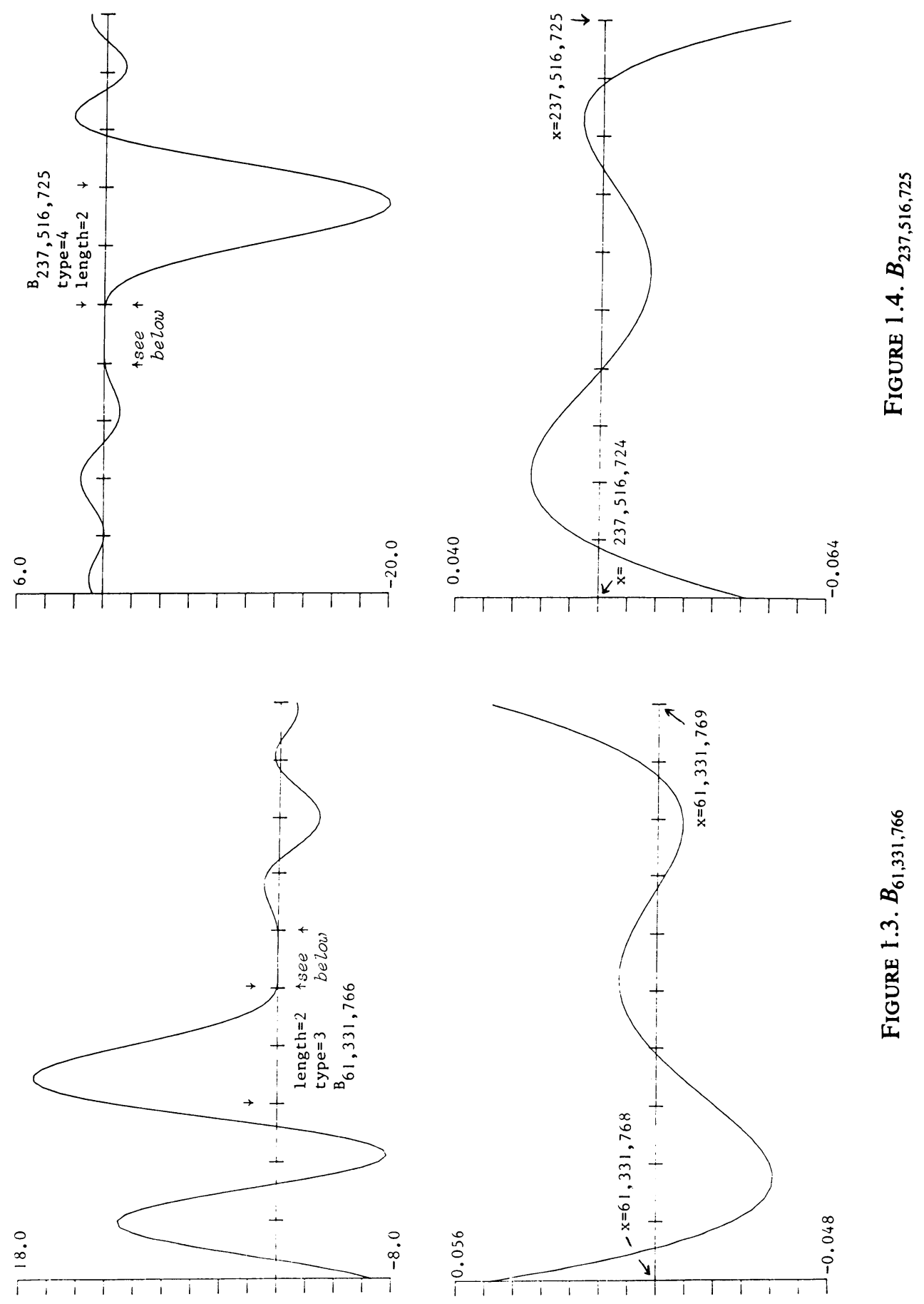
ZEROS OF THE RIEMANN ZETA FUNCTION

765
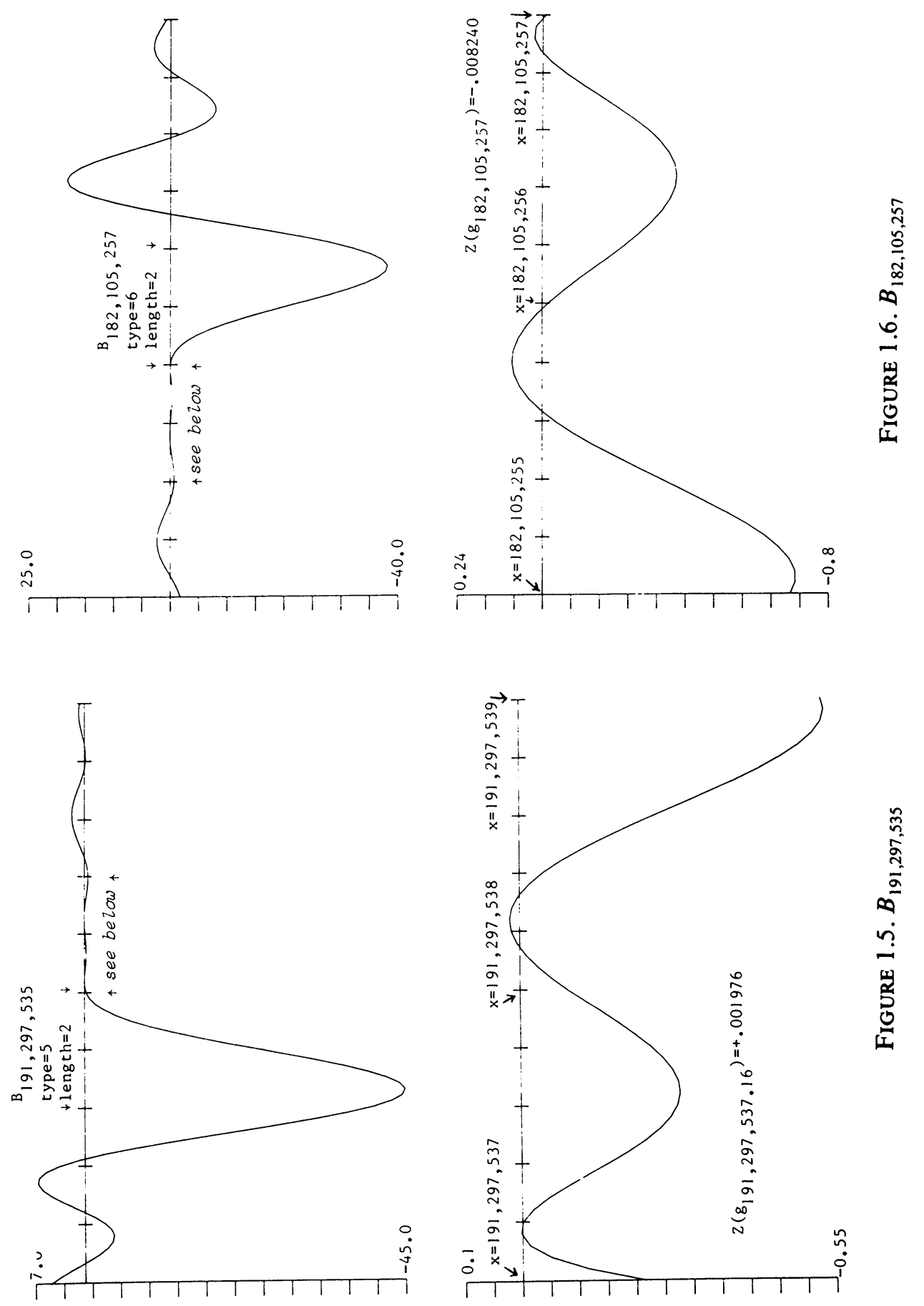

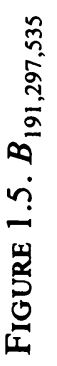



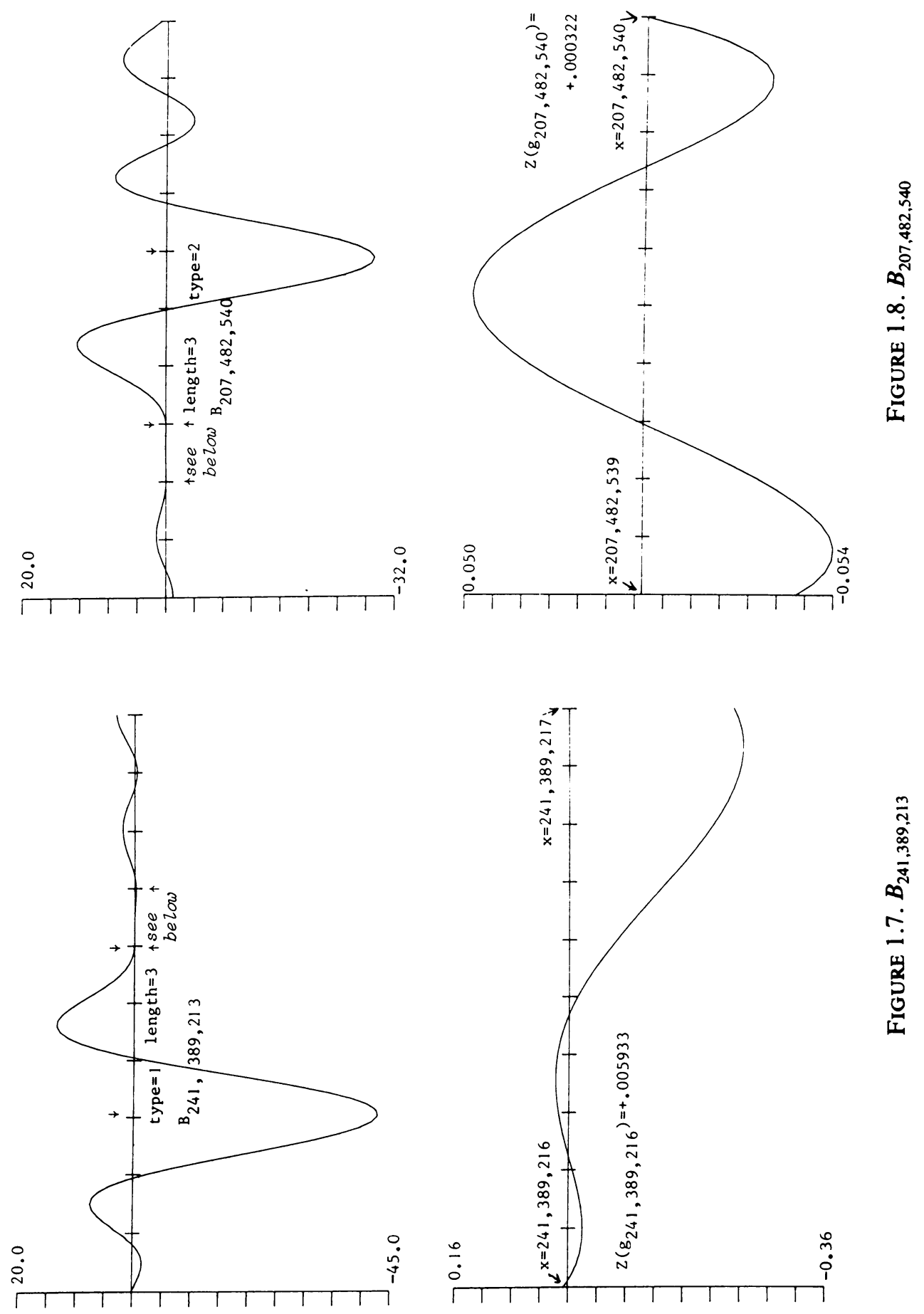
Table 3 gives the numbers of Gram blocks of length $\leqslant 8$ in $\left[g_{200,000,000}, g_{300,000,000}\right)$ for strings of $10^{7}$ successive zeros. The last line gives the totals for the whole range. The average Gram block length for this range is 1.2039, against 1.2003 and 1.1900 for the ranges $\left[g_{100,000,000}, g_{200,000,000}\right)$ and $\left[g_{0}, g_{100,000,000}\right)$, respectively. Note that the number of Gram blocks of length 1 is slowly decreasing in favor of the number of Gram blocks of lengths $\geqslant 2$ and $\leqslant 5$.

In Table 4 we present the number of Gram intervals $G_{j}=\left[g_{j}, g_{j+1}\right), n \leqslant j<n+$ $10^{7}$, which contain exactly $m$ zeros of $Z(t), 0 \leqslant m \leqslant 4$. Note that the number of Gram intervals with precisely one zero is slightly decreasing in favor of the number of Gram intervals with no zeros and those with 2 zeros.

In [1] and [2] we have listed the first occurrences of Gram blocks of various types. Here we have met only one new type of Gram block, viz. the type $(7,1)$-Gram block $B_{258,779,892}$.

In Table 5 we list the number of Gram blocks of type $(j, k), 1 \leqslant j \leqslant 8,1 \leqslant k \leqslant j$, in the range $\left[g_{200,000,000}, g_{300,000,000}\right)$. We also give the number of Gram blocks of length 2 with zero-pattern " 00 " and " 22 ", and those of length 3 with zero-pattern "0 10 ". The " 00 0"-blocks correspond to the 126 length 2-exceptions to Rosser's rule of types 1-6, and the " 2 2"-blocks correspond to the length 2-exceptions of types 5 and 6. The " 010 "-blocks correspond to the 3 (newly introduced) length 3 -exceptions of types 1 and 2 . The entries in parentheses denote the percentages with respect to the total number of blocks of length $j$, given in the final column. These percentages are nearly the same as those given in the corresponding table for the range $\left[g_{156,800,000}, g_{200,000,000}\right)$ in [2], and we conclude that our strategy of dealing with Gram blocks of length $j \geqslant 2$ is successful for $2 \leqslant j \leqslant 5$.

In order to give the reader an impression of the erratic behavior of $Z(t)$, we give in Figures 1.1-1.8 graphs of $Z(t)$ in the neighborhood of the first (observed) exceptions to Rosser's rule of lengths 2 and 3 and of various types. We have plotted $Z\left(g_{x}\right)$ with $x$ as a continuous independent variable. The exceptional Gram block is marked by two arrows pointing downwards. The adjacent Gram block where the "missing two" zeros are situated is marked by two arrows pointing upwards. A magnification of the latter block is shown in an accompanying graph. Some "critical" values of $Z(t)$ are explicitly mentioned.

Mathematical Centre

Kruislaan 413

1098 SJ Amsterdam, The Netherlands

1. R. P. Brent, “On the zeros of the Riemann zeta function in the critical strip," Math. Comp., v. 33, 1979, pp. 1361-1372.

2. R. P. BRent, J. van De Lune, H. J. J. Te Riele \& D. T. Winter, "On the zeros of the Riemann zeta function in the critical strip. II," Math. Comp., v. 39, 1982, pp. 681-688.

3. E. Karkoschka \& P. Werner, "Einige Ausnahmen zur Rosserschen Regel in der Theorie der Riemannschen Zetafunktion," Computing, v. 27, 1981, pp. 57-69.

4. J. van de Lune, H. J. J. TE Riele \& D. T. Winter, Rigorous High Speed Separation of Zeros of Riemann's Zeta Function, Report NW 113/81, October 1981, Mathematical Centre, Amsterdam.

5. J. van de LUne \& H. J. J. TE Riele, Rigorous High Speed Separation of Zeros of Riemann's Zeta Function, II, Report NN 26/82, June 1982, Mathematical Centre, Amsterdam. 